\title{
Berry phase in graphene: a semiclassical perspective
}

\author{
Pierre Carmier and Denis Ullmo \\ Univ. Paris-Sud, LPTMS UMR 8626, 91405 Orsay Cedex, France and \\ CNRS, LPTMS UMR 8626, 91405 Orsay Cedex, France
}

(Dated: February 11, 2013)

\begin{abstract}
We derive a semiclassical expression for the Green's function in graphene, in which the presence of a semiclassical phase is made apparent. The relationship between this semiclassical phase and the adiabatic Berry phase, usually referred to in this context, is discussed. These phases coincide for the perfectly linear Dirac dispersion relation. They differ however when a gap is opened at the Dirac point. We furthermore present several applications of our semiclassical formalism. In particular we provide, for various configurations, a semiclassical derivation of the electron's Landau levels, illustrating the role of the semiclassical "Berry-like" phase.

PACS numbers: 73.22.Dj, 03.65.Sq, 03.65.Vf
\end{abstract}

\section{INTRODUCTION}

Graphene [1, 2], a two-dimensional carbon based material forming a honeycomb lattice, has attracted a lot of attention since its experimental isolation has been proved possible 3, 4]. It is a gapless semiconductor in which, near half filling, electrons behave like massless Dirac particles, obeying a linear dispersion relation. Among the unusual properties of this two-dimensional carbon material stand out very distinctive quantum Hall properties, and in particular the $\sqrt{n}$ dependence of the energy in terms of the Landau level number $n$, and the existence of a Landau level with zero energy, which is associated with the presence of a Berry phase [1, 5].

The existence of this Berry phase and its implications for the Landau levels have been discussed in many places in different contexts (see e.g. 5, 6, 7] ). The direct connection between the Berry phase and the observable quantities under discussion is however not always as transparent as one may wish, and situations where, either because of disorder, or because one would like to confine the electrons into a finite region of space, a position dependent electrostatic potential or mass term is introduced, are usually not addressed.

The aim in this paper is to revisit this question of Berry phase in graphene within a semiclassical, and more specifically semiclassical Green's function, perspective. For sake of clarity, our emphasis in this present work will be more in providing this new point of view, and we shall therefore mainly illustrate it with the discussion of the standard problem of the Landau levels of electrons in a perpendicular and uniform magnetic field. Even in this familiar framework, we shall see however that our semiclassical approach makes it possible to address some non-trivial questions, such as the role of the Berry phase in situations for which a small mass term has to be included, opening in this way a gap at the Dirac point.

This article is therefore organized as follows. In section II, we derive, following closely the formalism of Bolte and Keppeler 88], the expression for the semiclassical Green's function in graphene. In particular we discuss in details the origin of the term corresponding to the
Berry phase. These results are extended in section [II] to a bilayer of graphene. We furthermore provide both for the monolayer and the bilayer cases the expression of the Gutzwiller trace formula for the semiclassical density of states, valid when classical periodic orbits are isolated. As an illustration of the Green's function formalism, we then apply it in section IV to the computation of Landau levels for a graphene sheet in constant magnetic field. We will see in particular that the modifications brought in by, for instance, trigonal warping, are easily included within our semiclassical formalism. We then come back in section V] to the discussion of the relationship between the semiclassical "Berry-like" phase obtained in our approach and the adiabatic Berry phase [9] usually discussed in this context.

\section{SEMICLASSICAL GREEN'S FUNCTION FOR GRAPHENE}

Starting from a tight-binding nearest neighbor model, the graphene Hamiltonian at low energies can be obtained by expanding the momentum near the Dirac points $\mathbf{K}$ and $\mathbf{K}^{\prime}$ of the Brillouin zone. For pure graphene, one obtains in this way in momentum representation [10, 11, 12, 13]

$$
\mathcal{H}_{g}^{0}=v_{F}\left(\alpha \sigma_{x} p_{x}+\sigma_{y} p_{y}\right)=v_{F}\left(\begin{array}{cc}
0 & \alpha p_{x}-i p_{y} \\
\alpha p_{x}+i p_{y} & 0
\end{array}\right)
$$

where the matrix structure originates from the existence of two sub-lattices (denoted $A$ and $B$ below) in the graphene honeycomb structure. In this equation, $v_{F}=3 t a /(2 \hbar)$ is the Fermi velocity, with $t$ the hopping parameter and $a$ the lattice constant, $\alpha$ is the valley index $(\alpha= \pm 1)$ labelling the two inequivalent points $\mathbf{K}$ and $\mathbf{K}^{\prime}$ in the Brillouin zone (not to be confused with the sublattice index), $\mathbf{p}$ is the momentum measured from these points, and $\sigma_{x, y}$ are Pauli matrices. This linear approximation to the graphene Hamiltonian will be valid as long as the condition $|\mathbf{p}| \ll \hbar / a$ is fulfilled.

We are interested here in a more general situation than 
the one of pure graphene, and would like to consider the case where, because of either disorder or the need to confine the electrons in some part of the graphene sheet, an electrostatic potential $U(\mathbf{r})$ and/or a [possibly position dependent] mass $m(\mathbf{r})$ have to be taken into account. We will not consider however tunneling contributions related to the Klein paradox, or boundary effects that may occur at the (zigzag, armchair, or generic) edges of the graphene sample. The graphene Hamiltonian then takes the more general form

$$
\mathcal{H}_{g}=v_{F}\left(\alpha \sigma_{x} \hat{\Pi}_{x}+\sigma_{y} \hat{\Pi}_{y}\right)+U(\mathbf{r}) \cdot \mathbf{1}_{2}+m(\mathbf{r}) v_{F}^{2} \sigma_{z}
$$

in which the magnetic field $\mathbf{B}(\mathbf{r})=\nabla \times \mathbf{A}(\mathbf{r})$ (if any) is taken into account by the Peierls substitution

$$
\hat{\mathbf{p}} \rightarrow \hat{\boldsymbol{\Pi}}=\hat{\mathbf{p}}+e \mathbf{A}(\mathbf{r})
$$

with $\mathbf{A}(\mathbf{r})$ the vector potential and $\hat{\mathbf{p}} \equiv-i \hbar \frac{\partial}{\partial \mathbf{r}}$.

For this problem, the Green's function $G\left(\mathbf{r}^{\prime \prime}, \mathbf{r}^{\prime}\right)$ is actually a $2 \times 2$ matrix defined by the differential equation

$$
\left(E . \mathbf{1}_{2}-\mathcal{H}_{g}\right) G\left(\mathbf{r}^{\prime \prime}, \mathbf{r}^{\prime} ; E\right)=\delta\left(\mathbf{r}^{\prime \prime}-\mathbf{r}^{\prime}\right) . \mathbf{1}_{2}
$$

(where $\mathcal{H}_{g}$ is applied to the variable $\mathbf{r}^{\prime \prime}$ ). To obtain a semiclassical solution of this equation, we shall proceed in two steps. First, assuming $\mathbf{r}^{\prime \prime}$ is far from the source location $\mathbf{r}^{\prime}$, we solve semiclassically (i.e. in the WKB approximation) the Schrödinger equation

$$
\left(E . \mathbf{1}_{2}-\mathcal{H}_{g}\right) G=0 \text {. }
$$

In a second stage we match this general solution to the exact Green's function of the "free" (i.e. with constant potential and mass) problem, valid near the singularity $\mathbf{r}^{\prime}$. We proceed now with this derivation.

\section{A. Far from the singularity: the WKB approximation}

Following [8], we seek a semiclassical solution of eq. (4) with $G$ of the form

$$
G\left(\mathbf{r}^{\prime \prime}, \mathbf{r}^{\prime} ; E\right)=\Gamma\left(\mathbf{r}^{\prime \prime}, \mathbf{r}^{\prime}\right) \exp \left[\frac{i}{\hbar} S\left(\mathbf{r}^{\prime \prime}, \mathbf{r}^{\prime}\right)\right]
$$

where $\Gamma$ is a $2 \times 2$ matrix. To lighten the notation, we drop for now the explicit dependence in the source position $\mathbf{r}^{\prime}$. Inserting (6) into (5) and expanding in $\hbar$ the resulting expression, we obtain at order $O\left(\hbar^{0}\right)$

$$
\left(E . \mathbf{1}_{2}-H\left(\frac{\partial S}{\partial \mathbf{r}^{\prime \prime}}, \mathbf{r}^{\prime \prime}\right)\right) \Gamma\left(\mathbf{r}^{\prime \prime}\right)=0
$$

and at order $O\left(\hbar^{1}\right)$

$$
\frac{\partial H}{\partial \mathbf{p}} \cdot \frac{\partial}{\partial \mathbf{r}^{\prime \prime}} \Gamma\left(\mathbf{r}^{\prime \prime}\right)=v_{F}\left(\alpha \sigma_{x} \frac{\partial}{\partial x^{\prime \prime}}+\sigma_{y} \frac{\partial}{\partial y^{\prime \prime}}\right) \Gamma\left(\mathbf{r}^{\prime \prime}\right)=0
$$

where $H(\mathbf{p}, \mathbf{r})$ is the classical symbol associated with the quantum Hamiltonian $\mathcal{H}_{g}$.
This classical Hamiltonian can be diagonalized, with the eigenvalues

$$
H^{ \pm}(\mathbf{p}, \mathbf{r})=U(\mathbf{r}) \pm \sqrt{m^{2}(\mathbf{r}) v_{F}^{4}+v_{F}^{2} \mathbf{\Pi}^{2}}
$$

and the corresponding normalized eigenvectors $V^{ \pm}(\mathbf{p}, \mathbf{r})$ (whose explicit expressions are given in appendix A). Writing the matrix $\Gamma\left(\mathbf{r}^{\prime \prime}\right)$ as $\left[V^{ \pm}\left(\frac{\partial S}{\partial \mathbf{r}^{\prime \prime}}, \mathbf{r}^{\prime \prime}\right) \cdot \tilde{\Gamma}^{ \pm}\left(\mathbf{r}^{\prime \prime}\right)\right]$, with $\tilde{\Gamma}^{ \pm}$a $1 \times 2$ matrix, the order $\hbar^{0}$ equation becomes

$$
E-H^{ \pm}\left(\frac{\partial S}{\partial \mathbf{r}^{\prime \prime}}, \mathbf{r}^{\prime \prime}\right)=0
$$

where the \pm sign must be taken according to the sign of $E-U\left(\mathbf{r}^{\prime \prime}\right)$.

Eq. (10) is the usual scalar Hamilton-Jacobi equation, which can be solved by the method of characteristics [14]. This amounts to constructing a 2-dimensional Lagrangian manifold $\mathcal{L}$ (in the 3 -dimensional energy surface in phase space) built as a 1-parameter family of trajectories following the classical equations of motion

$$
\begin{aligned}
\dot{\mathbf{r}} & =\frac{\partial H^{ \pm}}{\partial \mathbf{p}}(\mathbf{p}, \mathbf{r}) \\
\dot{\mathbf{p}} & =-\frac{\partial H^{ \pm}}{\partial \mathbf{r}}(\mathbf{p}, \mathbf{r})
\end{aligned}
$$

Given any such manifold, the action $S\left(\mathbf{r}^{\prime \prime}\right)=\int^{\mathbf{r}^{\prime \prime}} \mathbf{p} d \mathbf{r}$, where the integral is taken on an arbitrary path on $\mathcal{L}$, is a solution of (10).

The specific Lagrangian manifold that will correspond to the proper boundary conditions for $G\left(\mathbf{r}^{\prime \prime}, \mathbf{r}^{\prime}\right)$ near the source $\mathbf{r}^{\prime}$ is the one obtained from the trajectories leaving $\mathbf{r}^{\prime}$ with an arbitrary initial momentum $\mathbf{p}^{\prime}$ at energy $E$ :

$$
\begin{aligned}
\mathcal{L}^{ \pm}= & \{(\mathbf{p}(t), \mathbf{r}(t)), t \in[0, \infty) \\
& \text { such that } \left.\mathbf{r}(0)=\mathbf{r}^{\prime}, H^{ \pm}(\mathbf{p}(0), \mathbf{r}(0))=E\right\}
\end{aligned}
$$

(each point on the manifold is therefore parameterized by the time $t$ and the initial momentum $\mathbf{p}(0))$. The corresponding action can then be expressed as

$$
S^{ \pm}\left(\mathbf{r}^{\prime \prime}, \mathbf{r}^{\prime}\right)=\int_{\mathbf{r}^{\prime}}^{\mathbf{r}^{\prime \prime}} \mathbf{p} \cdot \dot{\mathbf{r}} d t
$$

along a trajectory $(\mathbf{p}(t), \mathbf{r}(t))$ joining $\mathbf{r}^{\prime}$ to $\mathbf{r}^{\prime \prime}$ at energy E.

Having obtained a solution of the $O\left(\hbar^{0}\right)$ equation, the prefactor $\tilde{\Gamma}$ is then determined by the $O\left(\hbar^{1}\right)$ equation (8), which, after multiplication on the left by $V^{ \pm \dagger}\left(\frac{\partial S^{ \pm}}{\partial \mathbf{r}^{\prime \prime}}, \mathbf{r}^{\prime \prime}\right)$, can be expressed as $\square \tilde{\Gamma}^{ \pm}=0$, where

$$
\square \equiv\left(V^{ \pm \dagger}\left(\frac{\partial S^{ \pm}}{\partial \mathbf{r}^{\prime \prime}}, \mathbf{r}^{\prime \prime}\right) \frac{\partial H}{\partial \mathbf{p}} \cdot \frac{\partial}{\partial \mathbf{r}^{\prime \prime}}\right) V^{ \pm}\left(\frac{\partial S^{ \pm}}{\partial \mathbf{r}^{\prime \prime}}, \mathbf{r}^{\prime \prime}\right) .
$$

The operator $\square$ can be decomposed as $\square=\square_{(1)}+\square_{(2)}$ with

$$
\square_{(1)}=\left(V^{ \pm \dagger} \frac{\partial H}{\partial \mathbf{p}} V^{ \pm}\right) \cdot \frac{\partial}{\partial \mathbf{r}^{\prime \prime}}
$$


and

$$
\square_{(2)}=V^{ \pm \dagger} \frac{\partial H}{\partial \mathbf{p}} \cdot\left(\frac{\partial V^{ \pm}}{\partial \mathbf{r}^{\prime \prime}}\right) .
$$

Noting that first order perturbation theory implies $V^{ \pm \dagger}(\partial H / \partial \mathbf{p}) V^{ \pm}=\left(\partial H^{ \pm} / \partial \mathbf{p}\right)$, one has straightforwardly that

$$
\square_{(1)}=\frac{\partial H^{ \pm}}{\partial \mathbf{p}} \cdot \frac{\partial}{\partial \mathbf{r}^{\prime \prime}}
$$

and that

$$
\operatorname{Re}\left(\square_{(2)}\right)=\frac{1}{2} \frac{\partial}{\partial \mathbf{r}^{\prime \prime}} \cdot\left(V^{ \pm \dagger} \frac{\partial H}{\partial \mathbf{p}} V^{ \pm}\right)=\frac{1}{2} \frac{\partial}{\partial \mathbf{r}^{\prime \prime}} \cdot \frac{\partial H^{ \pm}}{\partial \mathbf{p}} .
$$

(Note here that with respect to spatial derivation, $H^{ \pm} \equiv$ $\left.H^{ \pm}\left(\mathbf{r}^{\prime \prime}\right)=H^{ \pm}\left(\left(\partial S^{ \pm} / \partial \mathbf{r}^{\prime \prime}\right), \mathbf{r}^{\prime \prime}\right)\right)$. One recovers in this way, for the real part of $\square$, the usual expression valid for a scalar quantum system [14], which is expected since it basically expresses the conservation of probability.

The imaginary part of $\left(\square_{(2)}\right)$ however is not constrained by such a conservation law, as it affects only the phase of $\tilde{\Gamma}$, but encodes information about the adiabatic variation of the eigenvector $V^{ \pm}$along the followed trajectory. It needs therefore to be computed from the explicit expressions of the eigenvector and eigenvalues of $H(\mathbf{p}, \mathbf{r})$. The details of the algebra are given in appendix A. One obtains

$$
\left(\frac{\partial H^{ \pm}}{\partial \mathbf{p}} \cdot \frac{\partial}{\partial \mathbf{r}^{\prime \prime}}+\frac{1}{2} \frac{\partial}{\partial \mathbf{r}^{\prime \prime}} \cdot \frac{\partial H^{ \pm}}{\partial \mathbf{p}}+i M^{ \pm}\right) \tilde{\Gamma}^{ \pm}=0
$$

with

$$
\begin{aligned}
M^{ \pm}= & \frac{\alpha v_{F}^{2}}{2\left(E-U\left(\mathbf{r}^{\prime \prime}\right)\right)}(e \mathbf{B}+ \\
& \left.\frac{\Pi \times \frac{\partial}{\partial \mathbf{r}^{\prime \prime}}\left(m\left(\mathbf{r}^{\prime \prime}\right) v_{F}^{2}-U\left(\mathbf{r}^{\prime \prime}\right)\right)}{m\left(\mathbf{r}^{\prime \prime}\right) v_{F}^{2}+E-U\left(\mathbf{r}^{\prime \prime}\right)}\right) . \mathbf{e}_{z}
\end{aligned}
$$

( $\mathbf{e}_{z}$ is the unit vector in the direction perpendicular to the graphene sheet).

In the absence of the complex term $i M$, the scalar transport equation $\left(\frac{\partial H^{ \pm}}{\partial \mathbf{p}} \cdot \frac{\partial}{\partial \mathbf{r}^{\prime \prime}}+\frac{1}{2} \frac{\partial}{\partial \mathbf{r}^{\prime \prime}} \cdot \frac{\partial H^{ \pm}}{\partial \mathbf{p}}\right) \gamma^{ \pm}=0$ has the usual solution [14]

$$
\begin{aligned}
\gamma^{ \pm} & =C \frac{\exp \left(-i \frac{\pi}{2} \mu^{ \pm}\right)}{\sqrt{\left|\mathrm{J}^{ \pm}\left(\mathbf{r}^{\prime \prime}, \mathbf{r}^{\prime}\right)\right|}} \\
J^{ \pm}\left(\mathbf{r}^{\prime \prime}, \mathbf{r}^{\prime}\right) & =-\dot{r}_{\|}^{\prime \prime} \dot{r}_{\|}^{\prime}\left(\frac{\partial^{2} S^{ \pm}}{\partial r_{\perp}^{\prime \prime} \partial r_{\perp}^{\prime}}\right)^{-1} \\
& =\dot{r}_{\|}^{\prime \prime} \dot{r}_{\|}^{\prime}\left(\frac{\partial r_{\perp}^{\prime \prime}}{\partial p_{\perp}^{\prime}}\right)
\end{aligned}
$$

where $r_{\|}$and $r_{\perp}$ are the coordinates parallel and transverse to the trajectory (actually Eq. (20) remains valid for any system of coordinates) and $\mu^{ \pm}$is the Maslov index counting the (algebraic) number of caustic points. Writing

$$
\tilde{\Gamma}^{ \pm}=\gamma^{ \pm} \Sigma^{ \pm}
$$

we obtain that

$$
\left(\frac{\partial H^{ \pm}}{\partial \mathbf{p}} \cdot \frac{\partial}{\partial \mathbf{r}^{\prime \prime}}+i M^{ \pm}\right) \Sigma^{ \pm} \equiv\left(\frac{d}{d t}+i M^{ \pm}\right) \Sigma^{ \pm}=0
$$

and therefore $\Sigma^{ \pm}(t)=\exp \left(i \xi_{\mathrm{sc}}\right) \Sigma^{ \pm}(t=0)$, with

$$
\xi_{\mathrm{sc}}=-\int_{0}^{t} M^{ \pm}\left(\mathbf{p}\left(t^{\prime}\right), \mathbf{r}\left(t^{\prime}\right)\right) d t^{\prime}
$$

Summing the contributions corresponding to different orbits $j$ joining $\mathbf{r}^{\prime}$ to $\mathbf{r}^{\prime \prime}$ we get

$$
\begin{aligned}
& G\left(\mathbf{r}^{\prime \prime}, \mathbf{r}^{\prime} ; E\right)=\sum_{j: \mathbf{r}^{\prime} \rightarrow \mathbf{r}^{\prime \prime}} \gamma_{j}^{ \pm} V_{j}^{ \pm}\left(\mathbf{r}^{\prime \prime}\right) \Sigma_{j}^{ \pm}(t=0) \\
& \quad \exp \left(\frac{i}{\hbar} S_{j}^{ \pm}\left(\mathbf{r}^{\prime \prime}, \mathbf{r}^{\prime}\right)-i \int_{0}^{t_{j}} M_{j}^{ \pm}\left(\mathbf{p}\left(t^{\prime}\right), \mathbf{r}\left(t^{\prime}\right)\right) d t^{\prime}\right),
\end{aligned}
$$

where $V_{j}^{ \pm}\left(\mathbf{r}^{\prime \prime}\right) \equiv V^{ \pm}\left(\partial S_{j}^{ \pm} / \partial \mathbf{r}^{\prime \prime}, \mathbf{r}^{\prime \prime}\right)$ (and therefore depends not only on $\mathbf{r}^{\prime \prime}$ but also on the final momentum $\mathbf{p}_{j}^{\prime \prime}$ of the trajectory $j$ ).

The semiclassical phase $\xi_{\mathrm{sc}}$ Eq. (21) is the analog, in our context, of a Berry phase [9]. In the same way, it has its origin in the adiabatic change of the eigenvectors of the "internal degree of freedom" Hamiltonian $H(\mathbf{p}(\mathbf{r}), \mathbf{r})$ along the classical paths contributing to the semiclassical Green's function. Furthermore, in some circumstances, $\xi_{\text {sc }}$ exactly corresponds to the genuine Berry phase $\xi_{\text {ad }}$ defined for the adiabatic motion along the trajectory. This will be the case in particular for "pure" (i.e. without mass term) graphene. In general, however, $\xi_{\text {sc }}$ and $\xi_{\text {ad }}$ differ 15, 16]. We will come back to this point in section $[\mathrm{V}$ and in particular clarify the question of which of the two phases is relevant for the Landau levels.

\section{B. Matching to the exact solution near the source}

Sufficiently close, on the classical scale, to the source $\mathbf{r}^{\prime}$, we can neglect the variation of the various potentials and of the mass, i.e. assume $U(\mathbf{r})=U_{0}, m(\mathbf{r})=m_{0}$ and $\mathbf{A}(\mathbf{r})=0$. In this case we have the expression for the exact retarded Green's function:

$$
G=\left(\begin{array}{ll}
G_{A A} & G_{A B} \\
G_{B A} & G_{B B}
\end{array}\right)
$$

with 


$$
\begin{aligned}
& G_{A A}\left(\mathbf{r}^{\prime \prime}, \mathbf{r}^{\prime}, E+i \epsilon\right)=\left(-i \frac{m_{0} v_{F}^{2}+\sqrt{\zeta^{2}+m_{0}^{2} v_{F}^{4}}}{4\left(\hbar v_{F}\right)^{2}}\right) H_{0}\left(\frac{\zeta}{\hbar v_{F}}\left|\mathbf{r}^{\prime \prime}-\mathbf{r}^{\prime}\right|\right) \\
& G_{A B}\left(\mathbf{r}^{\prime \prime}, \mathbf{r}^{\prime}, E+i \epsilon\right)=\left(\alpha \frac{\zeta e^{-i \alpha \phi}}{4\left(\hbar v_{F}\right)^{2}}\right) H_{1}\left(\frac{\zeta}{\hbar v_{F}}\left|\mathbf{r}^{\prime \prime}-\mathbf{r}\right|\right)
\end{aligned}
$$

and $G_{B B}=G_{A A}\left(m_{0} \rightarrow-m_{0}\right), G_{B A}=G_{A B}(\phi \rightarrow-\phi)$. Here $\zeta=\sqrt{\left(E+i \epsilon-U_{0}\right)^{2}-m_{0}^{2} v_{F}^{4}}, \phi$ is the phase of $p_{x}+i p_{y}$ and $H_{0}$ and $H_{1}$ are Hankel functions of order 0 and 1. Asymptotically, as $\left|\mathbf{r}^{\prime \prime}-\mathbf{r}^{\prime}\right| \rightarrow+\infty, G_{A A}$ and $G_{A B}$ take the form

$$
G_{A A} \simeq-i \frac{m_{0} v_{F}^{2}+E-U_{0}}{4\left(\hbar v_{F}\right)^{2}} \sqrt{\frac{2}{\pi}} \frac{e^{i\left(k\left|\mathbf{r}^{\prime \prime}-\mathbf{r}^{\prime}\right|-\frac{\pi}{4}\right)}}{\sqrt{k\left|\mathbf{r}^{\prime \prime}-\mathbf{r}^{\prime}\right|}}
$$

$G_{A B} \simeq-i \alpha e^{-i \alpha \phi} \frac{\sqrt{\left(E-U_{0}\right)^{2}-m_{0}^{2} v_{F}^{4}}}{4\left(\hbar v_{F}\right)^{2}} \sqrt{\frac{2}{\pi}} \frac{e^{i\left(k\left|\mathbf{r}^{\prime \prime}-\mathbf{r}^{\prime}\right|-\frac{\pi}{4}\right)}}{\sqrt{k\left|\mathbf{r}^{\prime \prime}-\mathbf{r}^{\prime}\right|}}$

with $\hbar k=\frac{1}{v_{F}} \sqrt{\left(E-U_{0}\right)^{2}-m_{0}^{2} v_{F}^{4}}=|\mathbf{p}|$.

Let us assume $E-U_{0} \geq 0$, so that semiclassically we consider the positive eigenspace $H^{+}$. We note first that, in the free case considered here, the choice of the Lagrangian manifold $\mathcal{L}^{+}$given by (11) corresponds to the action $S^{+}\left(\mathbf{r}^{\prime \prime}, \mathbf{r}^{\prime}\right)=|\mathbf{p}| \cdot\left|\mathbf{r}^{\prime \prime}-\mathbf{r}^{\prime}\right|$ and to

$$
J^{+}\left(\mathbf{r}^{\prime \prime}, \mathbf{r}^{\prime}\right)=\frac{v_{F}^{4}}{\left(E-U\left(\mathbf{r}^{\prime}\right)\right)^{2}}|\mathbf{p}| \cdot\left|\mathbf{r}^{\prime \prime}-\mathbf{r}^{\prime}\right|,
$$

so that, as anticipated, the expression (22) matches the asymptotic expressions (25)-(26), provided one chooses $C=\frac{1}{\sqrt{2 i \pi \hbar}} \frac{1}{i \hbar}$ and

$$
\Sigma^{+}(t=0)=V^{+\dagger}\left(\frac{\partial S^{+}}{\partial \mathbf{r}^{\prime}}, \mathbf{r}^{\prime}\right) .
$$

The asymptotic expressions (25)-(26) are valid as soon as $\left|\mathbf{r}^{\prime \prime}-\mathbf{r}^{\prime}\right|$ is larger than a few Fermi wavelengths, which can still correspond to a distance short on the classical scale, and therefore such that the free Green's function is a good approximation. We can therefore use this matching condition to fix the prefactors $\Sigma^{+}(t=0)$ and $C$ in the generic case, obtaining finally

$$
\begin{aligned}
& G\left(\mathbf{r}^{\prime \prime}, \mathbf{r}^{\prime} ; E\right)=\frac{1}{\sqrt{2 i \pi \hbar}} \frac{1}{i \hbar} \\
& \sum_{j} \frac{\exp \left(\frac{i}{\hbar} S_{j}^{ \pm}-i \int_{0}^{t_{j}} M_{j}^{ \pm} d t^{\prime}-i \frac{\pi}{2} \mu_{j}^{ \pm}\right)}{\sqrt{\left|J_{j}^{ \pm}\right|}} V_{j}^{ \pm}\left(\mathbf{r}^{\prime \prime}\right) \cdot V_{j}^{ \pm \dagger}\left(\mathbf{r}^{\prime}\right) .
\end{aligned}
$$

\section{BILAYER GRAPHENE AND GUTZWILLER TRACE FORMULAE}

We turn now to a few extensions of the result derived in section II] We start with a generalization to the bi- layer graphene case, and then briefly discuss the resulting Gutzwiller trace formulae for the density of states, valid when classical periodic orbits are isolated in phase space (i.e., generically, for chaotic systems).

\section{A. Semiclassical Green's function for the bilayer case}

The bilayer graphene Hamiltonian can be written at low energy as [17]

$$
\mathcal{H}_{b i}^{0}=-\frac{1}{2 m^{*}}\left(\begin{array}{cc}
0 & \left(p_{x}-i p_{y}\right)^{2} \\
\left(p_{x}+i p_{y}\right)^{2} & 0
\end{array}\right)
$$

with $m^{*}=\gamma_{1} /\left(2 v_{F}^{2}\right)$, where $\gamma_{1}$ is the intra-layer coupling parameter. As before, we would like to include electric or magnetic fields, as well as a possibly position dependent mass term. We therefore consider the more general Hamiltonian

$$
\mathcal{H}_{b i}=U(\mathbf{r}) . \mathbf{1}_{2}+m(\mathbf{r}) v_{F}^{2} \sigma_{z}+\mathcal{H}_{b i}^{0}(\mathbf{p} \rightarrow \mathbf{\Pi}) .
$$

Following the same approach as above, one obtains the semiclassical Green's function in the form Eqs. (6) $-(22)$ except for a different expression of the classical Hamiltonian eigenenergies

$$
H^{ \pm}=U(\mathbf{r}) \pm \sqrt{m(\mathbf{r})^{2} v_{F}^{4}+\left(\frac{\boldsymbol{\Pi}^{2}}{2 m^{*}}\right)^{2}}
$$

and of the semiclassical ("Berry-like") phase term

$$
\begin{aligned}
M^{ \pm} & =\frac{1}{m^{*}} \sqrt{1-\frac{m\left(\mathbf{r}^{\prime \prime}\right)^{2} v_{F}^{4}}{\left(E-U\left(\mathbf{r}^{\prime \prime}\right)\right)^{2}}} \\
& \left( \pm e \mathbf{B}+\frac{1}{2} \frac{\boldsymbol{\Pi} \times \partial\left[m\left(\mathbf{r}^{\prime \prime}\right) v_{F}^{2}-U\left(\mathbf{r}^{\prime \prime}\right)\right] / \partial \mathbf{r}^{\prime \prime}}{m\left(\mathbf{r}^{\prime \prime}\right) v_{F}^{2}+E-U\left(\mathbf{r}^{\prime \prime}\right)}\right) \cdot \mathbf{e}_{z} .
\end{aligned}
$$

In the free case $\left(m(\mathbf{r}) \equiv m_{0}, U(\mathbf{r}) \equiv U_{0}\right)$, the exact Green's function can be shown to behave asymptotically as $\left|\mathbf{r}^{\prime \prime}-\mathbf{r}^{\prime}\right| \rightarrow+\infty$ as

$$
\begin{aligned}
G_{A A} & \simeq \frac{-i m^{*}}{4 \hbar^{2}} \sqrt{\frac{m_{0} v_{F}^{2}+E-U_{0}}{-m_{0} v_{F}^{2}+E-U_{0}}} \sqrt{\frac{2}{\pi}} \frac{e^{i\left(k\left|\mathbf{r}^{\prime \prime}-\mathbf{r}^{\prime}\right|-\frac{\pi}{4}\right)}}{\sqrt{k\left|\mathbf{r}^{\prime \prime}-\mathbf{r}^{\prime}\right|}} \\
G_{\tilde{B} \tilde{B}} & =G_{A A}\left(m_{0} \rightarrow-m_{0}\right) \\
G_{A \tilde{B}} & \simeq \frac{i m^{*}}{4 \hbar^{2}} e^{-2 i \phi} \sqrt{\frac{2}{\pi}} \frac{e^{i\left(k\left|\mathbf{r}^{\prime \prime}-\mathbf{r}^{\prime}\right|-\frac{\pi}{4}\right)}}{\sqrt{k\left|\mathbf{r}^{\prime \prime}-\mathbf{r}^{\prime}\right|}} \\
G_{\tilde{B} A} & =G_{A \tilde{B}}(\phi \rightarrow-\phi)
\end{aligned}
$$


with $\phi$ the phase of $p_{x}+i p_{y}$. Matching the exact solution near the source to the semiclassical expression far from the source eventually gives the semiclassical Green's function as a sum over all trajectories $j$ joining $\mathbf{r}^{\prime}$ to $\mathbf{r}^{\prime \prime}$ under the classical Hamiltonian $H^{+}$or $H^{-}$(depending on the sign of $\left.\left(E-U\left(\mathbf{r}^{\prime}\right)\right)\right)$

$$
\begin{gathered}
G\left(\mathbf{r}^{\prime \prime}, \mathbf{r}^{\prime} ; E\right)=\frac{1}{\sqrt{2 i \pi \hbar}} \frac{1}{i \hbar} \sum_{j} V_{j}^{ \pm}\left(\mathbf{r}^{\prime \prime}\right) V_{j}^{ \pm \dagger}\left(\mathbf{r}^{\prime}\right) \\
\frac{\exp \left(\frac{i}{\hbar} S_{j}^{ \pm}-i \int_{0}^{t_{j}} M_{j}^{ \pm} d t^{\prime}-i \frac{\pi}{2} \mu_{j}^{ \pm}\right)}{\sqrt{\left|J_{j}^{ \pm}\right|}}
\end{gathered}
$$

with $J^{ \pm}$given by Eq. (20).

\section{B. Trace formulae for isolated orbits}

One important application of the semiclassical expressions for the Green's functions is that, by taking their trace, one obtains a semiclassical approximation for the density of states $\rho(E)=\sum_{i} \delta\left(E-E_{i}\right)$. We have in mind here a quantum dot defined in a finite region of a graphene sheet (with the confinement imposed for instance through the mass term), and the $E_{i}$ are the corresponding discrete energies of the confined system. We will furthermore assume in this subsection the classical motion within the dot fully chaotic, so that all trajectories are isolated.

Starting from Eqs. (28) or (32), the semiclassical density of states can be obtained as the trace

$$
\rho(E) \equiv-\frac{1}{\pi} \operatorname{Im} \int d \mathbf{r} \operatorname{Tr}[G(\mathbf{r}, \mathbf{r} ; E)],
$$

(where $\operatorname{Tr}$ is the trace on the internal structure of the Green's function). The smooth (Weyl) part of the density of states, which is associated with "zero length" orbits, has the usual expression $\rho_{\text {Weyl }}(E)=\rho_{\text {Weyl }}^{+}(E)+\rho_{\text {Weyl }}^{-}(E)$ with

$$
\rho_{\mathrm{Weyl}}^{ \pm}(E)=\int \frac{d \mathbf{p} d \mathbf{r}}{(2 \pi \hbar)^{2}} \delta\left(E-H^{ \pm}(\mathbf{p}, \mathbf{r})\right)
$$

When potential and mass terms are constant this gives

$$
\rho_{\mathrm{Weyl}}^{ \pm}(E)=\frac{\left|E-U_{0}\right| \mathcal{A}}{2 \pi\left(\hbar v_{F}\right)^{2}} \Theta\left( \pm\left(E-U_{0}\right)-m_{0} v_{F}^{2}\right),
$$

with $\mathcal{A}$ the area of the graphene sheet and $\Theta$ the Heaviside step function.

The oscillating part $\rho_{\text {osc }}(E)$ of the density of states can then be obtained inserting the semiclassical expression for the Green's function in Eq. (33). Performing the integral on $\mathbf{r}$ in the stationary phase approximation imposes that, in the semiclassical sums Eqs. (28) or (32), only the trajectories with identical initial and final momentum should be kept. As a consequence, the sum over the index $j$ becomes a sum over periodic orbits.
In particular, in Eqs. (28) or (32), $V_{j}^{ \pm}\left(\mathbf{r}^{\prime \prime}\right)=V_{j}^{ \pm}\left(\mathbf{r}^{\prime}\right)$ since $\mathbf{r}^{\prime \prime}=\mathbf{r}^{\prime}=\mathbf{r}$ and $\mathbf{p}_{j}^{\prime \prime}=\mathbf{p}_{j}^{\prime}$ (remember that $V_{j}^{ \pm}(\mathbf{r}) \equiv V_{j}^{ \pm}\left(\mathbf{p}_{j}(\mathbf{r}), \mathbf{r}\right)$, so the second condition is necessary here). Therefore $\operatorname{Tr}\left[V_{j}^{ \pm}\left(\mathbf{r}^{\prime \prime}\right) \cdot V_{j}^{ \pm \dagger}\left(\mathbf{r}^{\prime}\right)\right]_{\mathbf{r}^{\prime \prime}=\mathbf{r}^{\prime}=\mathbf{r}}=1$. Once this point is recognized, the calculation of $\rho_{\text {osc }}$ from the semiclassical Green's functions is, up to the inclusion of the semiclassical "Berry-like" phase term $\oint_{i} M^{ \pm}(t) d t$, essentially the same as in the scalar case [18, 19] (see also the particularly clear discussion in 20$])$. We thus just quote the final results: $\rho(E)=\rho^{+}(E)+\rho^{-}(E)$; $\rho^{ \pm}(E)=\rho_{\text {Weyl }}^{ \pm}(E)+\rho_{\text {osc }}^{ \pm}(E)$, with

$$
\begin{aligned}
\rho_{\mathrm{osc}}^{ \pm}(E)= & \frac{1}{\pi \hbar} \sum_{\mathrm{p} . \mathrm{o} .} \frac{T_{\mathrm{ppo}}}{\sqrt{\left|\operatorname{det}\left(\tilde{M}_{\mathrm{po}}-1\right)\right|}} \\
& \cos \left(\frac{S_{\mathrm{po}}^{ \pm}}{\hbar}-\frac{\pi}{2} \sigma_{\mathrm{po}}^{ \pm}-\int_{0}^{T_{\mathrm{po}}} M^{ \pm} d t^{\prime}\right) .
\end{aligned}
$$

Here $\tilde{M}=\frac{\partial\left(p_{\perp}^{\prime \prime}, r_{\perp}^{\prime \prime}\right)}{\partial\left(p_{\perp}^{\prime}, r_{\perp}^{\prime}\right)}$ is the monodromy matrix, $\sigma^{ \pm}=\mu^{ \pm}+$ $\nu^{ \pm}$is the topologically invariant Maslov index $(\nu=0$ or 1 , depending on the sign of $d^{2} S_{j} / d r_{\perp}^{2}$, see the discussion in [20]), and $T_{\mathrm{ppo}}$ is the period of the primitive orbit $\left(T_{\mathrm{po}}=n T_{\mathrm{ppo}}\right.$ if the orbit consists of $n$ repetitions of the same path).

\section{GRAPHENE IN A CONSTANT MAGNETIC FIELD}

As an illustration of the semiclassical Green's function formalism, we consider in this section the simple (but useful) case of a graphene sheet immersed in a constant magnetic field, and show how some standard (and less standard) expressions can be easily re-obtained in this way. We start with the Landau levels in the monolayer and the bilayer, without potential or mass term $(U(\mathbf{r})=m(\mathbf{r})=0)$, and assuming the low-energy approximations Eqs. (1)-(29) of the Hamiltonian apply. We then study the influence of higher order corrections (e.g. trigonal warping) to this low-energy Hamiltonian. We finally consider the case where a finite mass term $m(\mathbf{r})=m_{0}=$ const., is introduced. This last example will be used to introduce the discussion on the distinction between the semiclassical and adiabatic Berry phases, with which we shall end this paper in the next section.

\section{A. Landau levels in monolayer graphene}

In the absence of confining potential or mass term, and with a constant magnetic field, the classical equations of motion in graphene are integrable and lead to cyclotronic motion, i.e. circular periodic orbits with period $T$ and 
radius $R$ given in the monolayer case by

$$
\begin{aligned}
T & =\frac{2 \pi}{v_{F}^{2}} \frac{E}{e B} \\
R & =\frac{v_{F}}{2 \pi} T .
\end{aligned}
$$

Since the periodic orbits are not isolated, we cannot use the Gutzwiller trace formula derived in the previous section and we have to obtain the density of states directly from inserting the semiclassical expression Eq. (28) in Eq. (33). Here however the classical dynamics is extremely simple: there is only one primitive orbit, and the sum over $j$ is actually a sum over the number of repetitions of this primitive circular orbit. We therefore have $S_{j}^{ \pm}=E t_{j} / 2$, with $t_{j}=j T$. Two caustics are furthermore traversed for each iteration of the orbit, one midway through the circle, the other when the orbit comes back to its starting point, and the Maslov index is thus $\mu_{j}^{ \pm}=2 j$ (note that, as discussed below, the last caustic should be included). Finally, the semiclassical "Berry-like" phase term Eq. (18) reduces here to $M_{j}^{ \pm}(\mathbf{r}(t))=\alpha v_{F}^{2} e B /(2 E)=$ const. so that

$$
\oint_{0}^{t_{j}} M_{j}^{ \pm}(t) d t=\alpha j \pi
$$

The only technical point in this calculation is therefore that since, whatever the initial momentum, all trajectories initiated in $\mathbf{r}^{\prime}=\mathbf{r}$ eventually return there, the final point $\mathbf{r}^{\prime \prime}=\mathbf{r}$ is a caustic $\left(\partial r_{\perp}^{\prime \prime} / \partial p_{\perp}^{\prime}=0\right)$ and the prefactor $1 / \sqrt{\left|J_{j}\right|}$ diverges. As discussed in the appendix $\mathrm{E}$ of 21], this divergence can be cured using a mixed representation of the Green's function, i.e. by expressing the Green's function $G\left(\mathbf{r}^{\prime \prime}, \mathbf{r}^{\prime}\right)$ in terms of its Fourier transform $\tilde{G}\left(p_{x}^{\prime \prime}, y^{\prime \prime} ; x^{\prime}, y^{\prime}\right)$ as

$$
\begin{aligned}
& G\left(x^{\prime \prime}, y^{\prime \prime} ; x^{\prime}, y^{\prime}\right)= \\
& \quad \frac{1}{\sqrt{-2 i \pi \hbar}} \int d p_{x}^{\prime \prime} \tilde{G}\left(p_{x}^{\prime \prime}, y^{\prime \prime} ; x^{\prime}, y^{\prime}\right) \exp \left(\frac{i}{\hbar} x^{\prime \prime} p_{x}^{\prime \prime}\right) .
\end{aligned}
$$

A semiclassical expression for $\tilde{G}$ can be derived in exactly the same way as for $G$, and leads to the same expression except for the transformations $S_{j} \rightarrow \tilde{S}_{j}=S_{j}-p_{x}^{\prime \prime} x^{\prime \prime}$ and $J_{j}=-\dot{y}^{\prime \prime} \dot{y}^{\prime}\left(\frac{\partial^{2} S_{j}}{\partial x^{\prime \prime} \partial x^{\prime}}\right)^{-1} \rightarrow \tilde{J}_{j}=-\dot{y}^{\prime \prime} \dot{y}^{\prime}\left(\frac{\partial^{2} \tilde{S}_{j}}{\partial p_{x}^{\prime \prime} \partial x^{\prime}}\right)^{-1}$. Thus

$$
\tilde{J}_{j}=\dot{y}^{\prime \prime} \dot{y}^{\prime}\left(\frac{\partial p_{x}^{\prime \prime}}{\partial p_{x}^{\prime}}\right)
$$

which is not diverging since for the cyclotron motion $\partial p_{x}^{\prime \prime} / \partial p_{x}^{\prime}=1$. The integral over $p_{x}^{\prime \prime}$ in (39) becomes then straightforward (noting that $d p_{x}^{\prime \prime} / \dot{y}^{\prime \prime}=d \theta$, with $\theta$ the angle made by the initial velocity with the $x$ axis, this integral basically provides a factor $\int_{0}^{2 \pi} d \theta=2 \pi$ ). Furthermore the integration over position in Eq. (33) amounts to a multiplication by the area $\mathcal{A}$ of the graphene sheet, and as in section IIIB, $\operatorname{Tr}\left[V_{j}^{ \pm}\left(\mathbf{r}^{\prime \prime}\right) \cdot V_{j}^{ \pm \dagger}\left(\mathbf{r}^{\prime}\right)\right]_{\mid \mathbf{r}^{\prime \prime}=\mathbf{r}^{\prime}=\mathbf{r}}=1$ since the final and initial momenta are identical. One therefore obtains

$$
\rho^{\mathrm{osc}}(E)=\frac{|E| \mathcal{A}}{\pi\left(\hbar v_{F}\right)^{2}} \sum_{j=1}^{+\infty} \cos 2 \pi j \frac{E^{2}}{2 \hbar e B v_{F}^{2}} .
$$

The total density of states is then $\rho(E)=\rho_{\text {Weyl }}(E)+$ $\rho^{\text {osc }}(E)$ with $\rho_{\text {Weyl }}(E)$ the smooth density of states (which is identical to the one without magnetic field) given by Eq. (34). Using the Poisson formula, we therefore have

$$
\rho(E)=\frac{\mathcal{A}}{2 \pi l_{B}^{2}} \sum_{n=-\infty}^{+\infty} \delta\left(E-E_{n}\right)
$$

with $l_{B}=\sqrt{\hbar /(e B)}$ and

$$
E_{n}=\operatorname{sign}(n) v_{F} \sqrt{|2 n \hbar e B|} .
$$

We recover in this way the expression of the Landau levels as obtained in a fully quantal derivation [12]. This approach furthermore provides a direct link between the phase $\oint_{0}^{t_{j}} M_{j}(t) d t=\alpha j \pi$ and the existence of a zero energy level, as it cancels out the phase associated with the Maslov indices (another example of such a cancellation can be found in 22]). An alternative semiclassical derivation of the graphene Landau levels can be obtained starting from the Dirac oscillator [23], in the limit of massless carriers, provided the frequency of the oscillator is taken to be the cyclotronic one.

\section{B. Landau levels in bilayer graphene}

Considering now the bilayer case, we can proceed in exactly the same way as above except for two differences. First, the period $T$ and radius $R$ are now given by

$$
\begin{aligned}
T & =\frac{2 \pi}{\omega}=2 \pi \frac{m^{*}}{e B} \\
R & =\sqrt{\frac{|E|}{2 \pi^{2} m^{*}}} T .
\end{aligned}
$$

Second, the semiclassical "Berry-like" phase term Eq. (31) now reduces to $M_{j}^{ \pm}(\mathbf{r}(t))= \pm e B / m^{*}=$ const., so that

$$
\oint_{0}^{t_{j}} M_{j}(t) d t=2 j \pi
$$

The Berry-like phase does not in this case compensate the phase associated with the Maslov index. Noting furthermore that, for the bilayer graphene, $\rho_{\text {Weyl }}(E)=$ $m^{*} \mathcal{A} /\left(2 \pi \hbar^{2}\right)$, we obtain

$$
\rho(E)=\frac{\mathcal{A}}{2 \pi l_{B}^{2}} \sum_{n=-\infty}^{+\infty} \delta\left(E-E_{n}^{\mathrm{sc}}\right)
$$


where

$$
E_{n}^{\mathrm{sc}}=\hbar \omega\left(n-\frac{1}{2}\right)
$$

is the semiclassical approximation to the exact quantum values of the Landau levels, $E_{n}{ }^{\text {quant }}=\hbar \omega \sqrt{n(n-1)}=$ $\hbar \omega\left(n-\frac{1}{2}\right)+O\left(\frac{1}{n}\right)$. The semiclassical calculation fails here to account for the $O\left(\frac{1}{n}\right)$ term. The $n=0$ and $n=1$ Landau levels, which both have zero energy, are therefore not correctly described within this semiclassical approach. However, for $n \geq 2$, the agreement between the semiclassical approximation and the exact result is quantitatively very good.

\section{Influence of higher order corrections (in the parameter $(a|\mathbf{p}| / \hbar))$}

The next example to which we shall apply our semiclassical formalism is the shift of the Landau levels associated with deviations, for large momenta, to the linear approximation of the graphene dispersion relation Eq. (1) [24].

Starting from a tight-binding description of the graphene monolayer in which the effect of the next-tonearest neighbor hopping is taken into account via the parameter $t^{\prime} \ll t$, and expanding the resulting dispersion relation near the $\mathbf{K}$ and $\mathbf{K}^{\prime}$ points up to third order in $(a|\mathbf{p}| / \hbar)$ (the reason for expanding up to third order will become clear below), the resulting Hamiltonian reads (in the absence of electric or magnetic fields) 24]

$$
\mathcal{H}_{g}^{\prime}=\mathcal{H}_{g}^{0}+\left(\begin{array}{cc}
h^{\prime}(\mathbf{p}) & h(\mathbf{p})^{*} \\
h(\mathbf{p}) & h^{\prime}(\mathbf{p})
\end{array}\right)
$$

with $\mathcal{H}_{g}^{0}$ given by Eq. (11) and

$$
\begin{aligned}
h^{\prime}(\mathbf{p}) & =-3 t^{\prime}+6 \frac{t^{\prime}}{t} v_{F}|\mathbf{p}|\left(\frac{v_{F}}{6 t}|\mathbf{p}|-2 \alpha\left(\frac{v_{F}}{6 t}\right)^{2} \mathbf{p}^{2} \cos 3 \phi_{\mathbf{p}}\right) \\
h(\mathbf{p}) & =-v_{F}\left(\frac{v_{F}}{6 t}\left(\alpha p_{x}-i p_{y}\right)^{2}+2\left(\frac{v_{F}}{6 t}\right)^{2} \mathbf{p}^{2}\left(\alpha p_{x}+i p_{y}\right)\right) .
\end{aligned}
$$

Keeping only terms no greater than third order in momentum, the eigenvalues of the associated classical Hamiltonian can be expressed as

$$
\begin{aligned}
& H^{ \pm}=h^{\prime}(\mathbf{p}) \pm v_{F}|\mathbf{p}|\left(1-\alpha \frac{v_{F}}{6 t}|\mathbf{p}| \cos 3 \phi_{\mathbf{p}}\right. \\
& \left.-\frac{1}{2}\left(\frac{v_{F}}{6 t}\right)^{2} \mathbf{p}^{2}\left(3+\cos ^{2} 3 \phi_{\mathbf{p}}\right)\right) \text {, }
\end{aligned}
$$

with $\phi_{\mathbf{p}}=\arctan \left(p_{y} / p_{x}\right)$. The anisotropic terms, proportional to $\cos 3 \phi_{\mathbf{p}}$, are often referred to as trigonal warping. Recall now this expansion is valid if the condition $|\mathbf{p}| \ll \hbar / a$ is fulfilled. Rewriting the expression $\left(v_{F} / 6 t\right)|\mathbf{p}|=|\mathbf{p}| a /(4 \hbar)$, higher order terms in $H^{ \pm}$can thus be viewed as a perturbation of the original eigenvalue $H^{ \pm}= \pm v_{F}|\mathbf{p}|$ in the small parameter $\left(\lambda v_{F}|\mathbf{p}|\right)$, where $\lambda \equiv \alpha / 6 t$ will be used below to identify the order in the perturbation. In the semiclassical limit $(\hbar \rightarrow 0)$, only the modification of the action needs to be taken into account since this latter is multiplied by the large parameter $1 / \hbar$. Our aim is therefore to compute the (first and second order here) corrections to the action in an expansion in $\lambda$

$$
S=S_{0}+\lambda \delta^{(1)} S+\lambda^{2} \delta^{(2)} S .
$$

In the presence of a constant magnetic field, the classical equations of motion derived from the first order approximation $H^{ \pm}= \pm v_{F}|\boldsymbol{\Pi}|$ are integrable, and this property is not modified by the addition of terms in $H^{ \pm}$ depending only on $|\boldsymbol{\Pi}|$. This can be easily shown by performing a canonical transformation to the guiding center coordinates. For sake of completeness, this canonical change of variables is detailed in appendix $B$, The new coordinates read

$$
\begin{aligned}
& \mathbf{R}=\left(\frac{1}{e B} \Pi_{y}, x_{0}\right) \\
& \mathbf{P}=\left(\Pi_{x}, e B y_{0}\right)
\end{aligned}
$$

with $\mathbf{r}_{0}$ the center of the cyclotron orbit, so that $|\boldsymbol{\Pi}|=$ $\sqrt{P_{x}^{2}+(e B X)^{2}}$ and $\tan \phi_{\Pi}=e B X / P_{x}$. We thus have

$$
H^{+}=-3 t^{\prime}+\rho-\mu_{2} \lambda \rho^{2}-\mu_{1} \lambda^{2} \rho^{3}
$$

with $v_{F}\left(P_{x}+i e B X\right)=\rho e^{i \phi}, \mu_{2}=\left(\cos 3 \phi-6 \alpha \frac{t^{\prime}}{t}\right)$, and $\mu_{1}=\frac{1}{2}\left(3+\cos ^{2} 3 \phi+6 \alpha \frac{t^{\prime}}{t} \cos 3 \phi\right)$. In this new system of coordinates, the action is easily calculated as

$$
S=\int \mathbf{P} d \mathbf{R}=\int P_{x} d X=\frac{1}{2 v_{F}^{2} e B} \int_{0}^{2 \pi} \rho^{2}(\phi) d \phi
$$

with the constraint $E=H^{+}$. Therefore, to order $\lambda^{2}$, and with $E^{\prime}=E+3 t^{\prime}$

$$
\rho^{2}=E^{\prime 2}+2 \mu_{1} \lambda E^{\prime 3}+\left(5 \mu_{1}^{2}+2 \mu_{2}\right) \lambda^{2} E^{\prime 4}
$$

which gives for the action

$$
\begin{aligned}
S=\frac{1}{2 v_{F}^{2} e B} & \left(2 \pi E^{\prime 2}-24 \pi \alpha \frac{t^{\prime}}{t} \lambda E^{\prime 3}\right. \\
+ & \left.12 \pi\left(1+30\left(\frac{t^{\prime}}{t}\right)^{2}\right) \lambda^{2} E^{\prime 4}\right) .
\end{aligned}
$$

The third order terms had to be taken into account in the low-energy expansion, since their contribution in the second order correction of the action is of the same magnitude as that of second order terms. The third order term in the next-to-nearest neighbor contribution however cancels out in the calculation of $S$ and thus a second order expansion in $h^{\prime}(\mathbf{p})$ would have been sufficient. Introducing this shift in the action in the Landau-levels calculation of section IVA finally gives

$$
\begin{aligned}
& E_{n}^{\prime}=E_{n}\left(1 \pm 6 \alpha \frac{t^{\prime}}{t} \lambda E_{n}-3 \lambda^{2} E_{n}^{2}\right) \\
& =E_{n}\left(1 \pm \frac{3 t^{\prime}}{\sqrt{2} t} \frac{a}{l_{B}} \sqrt{n}-\frac{3}{8}\left(\frac{a}{l_{B}}\right)^{2} n\right)
\end{aligned}
$$


$\left(l_{B}=\sqrt{\hbar /(e B)}\right.$ is the magnetic length). This result is identical to the one obtained purely quantum mechanically in 24]. As discussed in this paper, the resulting effect is however too small to interpret shift in Landau levels observed experimentally by Plochocka et al. 24] .

\section{Effect of a mass term}

To end this section, let us consider the effect of a constant mass term $m_{0} v_{F}^{2} \sigma_{z}$ in the graphene Hamiltonian, so that

$$
H^{ \pm}= \pm \sqrt{m_{0}^{2} v_{F}^{4}+v_{F}^{2} \Pi^{2}} .
$$

Interestingly, a constant mass term does not modify the time derivative $M(t)$ of the semiclassical Berry-like phase since (see Eq. (18)) it depends only on the gradient of $m(r)$. Furthermore, as shown by a direct calculation, the energy dependence of the Landau frequency is not affected either by the mass term. Therefore

$$
\begin{aligned}
T & =\frac{2 \pi}{\omega}=\frac{2 \pi}{v_{F}^{2}} \frac{E}{e B} \\
M(\mathbf{r}(t)) & =\alpha v_{F}^{2} \frac{e B}{2 E}=\text { const. }
\end{aligned}
$$

and the semiclassical phase

$$
\oint_{0}^{t_{j}} M_{j}(t) d t=j \alpha \pi
$$

is the same as without the mass term.

The $m_{0}$ dependence of the Landau level position is therefore entirely due to the $m_{0}$ variation of the action

$$
S_{j}=j \pi \frac{E^{2}}{e B v_{F}^{2}}\left[1-\left(\frac{m_{0} v_{F}^{2}}{E}\right)^{2}\right],
$$

which, following the same steps as in section IVA gives $\rho(E)=\left(\mathcal{A} /\left(2 \pi l_{B}^{2}\right)\right) \sum_{n=0}^{+\infty} \delta\left(E \pm E_{n}\right)$, with

$$
\begin{aligned}
E_{n} & =\sqrt{E_{n}^{2}(0)+m_{0}^{2} v_{F}^{4}} \\
& \simeq E_{n}(0)\left(1+\frac{1}{4 n} \frac{\left(m_{0} v_{F}\right)^{2}}{e \hbar B}\right)
\end{aligned}
$$

$\left(E_{n}(0)\right.$ is the value of $E_{n}$ at $m_{0}=0$ given by Eq. (43) $)$. One recovers semiclassically in this way the result originally derived by Haldane [25].

\section{SEMICLASSICAL VERSUS ADIABATIC BERRY PHASE}

We would like to finish this paper with some general discussion concerning the semiclassical phase

$$
\begin{aligned}
\xi_{\mathrm{sc}} & \equiv-\oint_{0}^{T} M_{\mathrm{sc}}(\mathbf{p}(t), \mathbf{r}(t)) d t \\
M_{\mathrm{sc}}(\mathbf{p}(t), \mathbf{r}(t)) & =\operatorname{Im}\left[V^{ \pm \dagger} \frac{\partial H}{\partial \mathbf{p}} \cdot\left(\frac{\partial V^{ \pm}}{\partial \mathbf{r}}\right)\right]
\end{aligned}
$$

(see Eq. (14) ) computed on a periodic orbit $(\mathbf{p}(t), \mathbf{r}(t))$ (of period $T$ ).

That, for a clean graphene monolayer without a mass term, $\xi_{\mathrm{sc}}=\mp \pi$ (as expressed by Eq. (38), with $j=1$ ) is usually said to be expected since the corresponding configuration is exactly the one discussed in detail by Berry in his 1984 paper [9]: the path of integration corresponds to encircling once the Dirac point, where the $H^{+}$and $H^{-}$manifolds intersect. This argument however relies on an exact intersection between the two manifolds, and should a priori not apply when a mass term $m_{0}$ introduces a gap. From this perspective, one does not expect the Berry phase to be equal to $\pm \pi$ when $m_{0} \neq 0$, and Eq. (56) may come as a surprise. (Note though this was already observed in 26].)

The resolution of this apparent paradox is that, as discussed in 15, 16], the semiclassical phase $\xi_{\text {sc }}$ defined by Eq. (21) and the adiabatic phase introduced by Berry are closely related, but eventually different, quantities. Both of them are induced by the adiabatic variation of the eigenstates $V^{+}$and $V^{-}$along the trajectory. However, the point of view taken in the semiclassical approach is that both the internal space (associated here with the sub-lattices $(A, B))$ and the external space (position $\mathbf{r}$ ) are coupled dynamical variables. Treating the coupling between these variables in the semiclassical approximation (which indeed implicitly assumes that the "external" variable is slow and the internal variable fast) leads to the semiclassical expression (61).

The problem Berry was considering in his seminal article [9] is however different: in that case, only the internal degree of freedom is considered a dynamical variable, and the external degrees of freedom are actually a space of parameters assumed to be entirely controlled by the experimentalist. One may in that case of course choose this path as the classical trajectory $(\mathbf{r}(t)$ ) (with $H(\mathbf{r}) \equiv H(\mathbf{p}(\mathbf{r}), \mathbf{r}))$ determined by the dynamics in the semiclassical approach. In that case however the corresponding phase is given by [9]

$$
\begin{aligned}
\xi_{\mathrm{ad}} & \equiv \oint_{0}^{T} M_{\mathrm{ad}}(\mathbf{r}(t)) d t \\
M_{\mathrm{ad}}(\mathbf{r}(t)) & =i V^{ \pm \dagger} \frac{\partial V^{ \pm}}{\partial \mathbf{r}} \cdot \dot{\mathbf{r}}
\end{aligned}
$$

(the normalization of $V^{ \pm}$ensures that $M_{\mathrm{ad}}$ is real).

Let us assume, for this discussion, that we are interested in the evolution of the eigenstate $V^{+}$associated with the positive eigenvalue $H^{+}$. Furthermore, let us switch to the bra/ket notation for the eigenvector and write $V^{ \pm} \equiv| \pm\rangle, V^{ \pm \dagger} \equiv\langle \pm|$. First order perturbation theory implies $\dot{\mathbf{r}}=\partial H^{+} / \partial \mathbf{p}=\langle+|(\partial H / \partial \mathbf{p})|+\rangle$, and therefore Eq. (63) can be rewritten as

$$
M_{\mathrm{ad}}(\mathbf{r}(t))=i\left\langle+\frac{\partial H}{\partial \mathbf{p}} \mid+\right\rangle \cdot\left\langle+\mid \frac{\partial}{\partial \mathbf{r}}+\right\rangle .
$$

On the other hand, inserting the identity $\mathbf{1}_{2}=|+\rangle\langle++$ 
$|-\rangle\langle-|$ in Eq. (61),

$M_{\mathrm{sc}}(\mathbf{r}(t))=\operatorname{Im}\left[\left\langle+\left|\frac{\partial H}{\partial \mathbf{p}}\right|+\right\rangle \cdot\left\langle+\frac{\partial}{\partial \mathbf{r}}+\right\rangle+\left\langle+\left|\frac{\partial H}{\partial \mathbf{p}}\right|-\right\rangle \cdot\left\langle-\mid \frac{\partial}{\partial \mathbf{r}}+\right\rangle\right]$.

Thus, the adiabatic and semiclassical phases actually differ from the quantity

$$
\xi_{\mathrm{ad}}-\xi_{\mathrm{sc}}=\oint_{0}^{T} \operatorname{Im}\left[\left\langle+\left|\frac{\partial H}{\partial \mathbf{p}}\right|-\right\rangle \cdot\left\langle-\mid \frac{\partial}{\partial \mathbf{r}}+\right\rangle\right] d t .
$$

From this expression, we can see that in the absence of a mass term, but for an arbitrary electrostatic potential $U(\mathbf{r})$, the semiclassical and Berry phases are identical. Indeed, for $m(\mathbf{r}) \equiv 0$, the expressions Eqs. (A2)- (A3) for the eigenvectors of $H(\mathbf{p}, \mathbf{r})$ take the simple form

$$
\begin{aligned}
& |+\rangle=\frac{1}{\sqrt{2}}\left(\begin{array}{c}
1 \\
\alpha e^{i \alpha \phi}
\end{array}\right) \\
& -\rangle=\frac{1}{\sqrt{2}}\left(\begin{array}{c}
\alpha e^{-i \alpha \phi} \\
-1
\end{array}\right),
\end{aligned}
$$

with $\phi$ the phase of $\Pi_{x}+i \Pi_{y}$. As a consequence

$$
\begin{aligned}
\left\langle H \frac{\partial H}{\partial \mathbf{p}} \mid-\right\rangle \cdot\left\langle-\mid \frac{\partial}{\partial \mathbf{r}}+\right\rangle & =\frac{v_{F}}{2}\left(-\sin \phi \partial_{x} \phi+\cos \phi \partial_{y} \phi\right)(69) \\
\left\langle H \frac{\partial H}{\partial \mathbf{p}} \mid+\right\rangle \cdot\left\langle H \frac{\partial}{\partial \mathbf{r}}+\right\rangle & =i \frac{\alpha v_{F}}{2}\left(\cos \phi \partial_{x} \phi+\sin \phi \partial_{y} \phi\right) \\
& =\frac{i \alpha}{2} \frac{d \phi}{d t} .
\end{aligned}
$$

The right hand side of Eq. (69) is purely real, implying that, in the simple case $m=0$ considered here, $\xi_{\text {ad }}-$ $\xi_{\mathrm{sc}}=0$. Eq. (70) then expresses that, independently of the nature of the electrostatic potential $U(\mathbf{r})$, the here identical - Berry phase and semiclassical phase are just given by plus or minus (depending on $\alpha$ ) half the angle of rotation of the velocity vector. In particular, as demonstrated by Berry from geometric arguments [9], we see here from a direct calculation that for a periodic orbit, $\xi_{\mathrm{ad}}=\xi_{\mathrm{sc}}=-\alpha j \pi$, with $j$ the number of windings of the trajectory. This makes particularly simple the inclusion of the semiclassical phase in the Gutzwiller trace formula Eq. (35) when $m=0$.

Similarly, for the bilayer Hamiltonian Eq. (30) with $m(\mathbf{r}) \equiv 0$, we have

$$
\begin{aligned}
& H\rangle=\frac{1}{\sqrt{2}}\left(\begin{array}{c}
1 \\
-e^{i 2 \phi}
\end{array}\right) \\
& H=\frac{1}{\sqrt{2}}\left(\begin{array}{c}
e^{-i 2 \phi} \\
1
\end{array}\right),
\end{aligned}
$$

with $\phi$ the phase of $\Pi_{x}+i \Pi_{y}$, and

$$
\begin{aligned}
\left\langle+\left|\frac{\partial H}{\partial \mathbf{p}}\right|-\right\rangle \cdot\left\langle-\mid \frac{\partial}{\partial \mathbf{r}}+\right\rangle & =\frac{|\Pi|}{m^{*}}\left(-\sin \phi \partial_{x} \phi+\cos \phi \partial_{y} \phi \gamma 73\right) \\
\left\langle H\left|\frac{\partial H}{\partial \mathbf{p}}\right|+\right\rangle \cdot\left\langle H \frac{\partial}{\partial \mathbf{r}}+\right\rangle & =i \frac{|\Pi|}{m^{*}}\left(\cos \phi \partial_{x} \phi+\sin \phi \partial_{y} \phi\right) \\
& =i \frac{d \phi}{d t}
\end{aligned}
$$

Again, the Berry phase and semiclassical phase are identical if $m(\mathbf{r}) \equiv 0$ (as Eq. (73) is purely real), and both phases are given by the angle of rotation of the velocity vector.

For both bilayer and monolayer graphene, it has to be born in mind however that in the generic case $m(\mathbf{r}) \neq 0$, the semiclassical phase $\xi_{\text {sc }}$ should in general differ from the Berry phase $\xi_{\text {ad }}$. Furthermore, we do not have a general argument constraining any of the two phases to be directly related to the winding of the velocity vector (beyond the case where both the mass and the electrostatic potential are constant).

\section{CONCLUSION}

To conclude, we have derived an expression for the semiclassical Green's function in graphene and discussed in particular the semiclassical phase associated with the internal pseudo-spin structure. If no mass term is included in the graphene Hamiltonian, this semiclassical phase is identical to the corresponding (adiabatic) Berry phase. In that case both phases are, up to a sign, given by half the angle of rotation of the velocity vector. For a bilayer of graphene, the same result holds but with a phase which is twice as large.

When a mass term is introduced however, the semiclassical and Berry phases in general differ. In particular, for a clean graphene sheet in a constant magnetic field, we have shown that the semiclassical phase remains unmodified upon the inclusion of a constant mass term $m(\mathbf{r})=m_{0}$, while the corresponding Berry phase $\xi_{\text {ad }}=\left[m_{0} /\left(E-U_{0}\right)-1\right] \alpha j \pi$ shows some dependence on $m_{0}$. We have shown furthermore that in this case, what is relevant to the calculation of the Landau levels is the semiclassical, rather than the Berry, phase. Other applications of our semiclassical formalism were also discussed, including the effect of higher order terms of the graphene Hamiltonian - e.g. trigonal warping - on the position of the Landau levels.

The semiclassical approximation to the graphene Green's function should prove a useful tool when considering confined electron systems in graphene, such as graphene nanoribbons, or more complicated geometries.

We have benefited from helpful discussions with E. Bogomolny, J.-N. Fuchs, M.-O. Goerbig, G. Montambaux and F. Piéchon, and thank as well all active participants of the weekly graphene "journal-club" held in LPS, Orsay.

\section{APPENDIX A: IMAGINARY PART OF THE OPERATOR $\left(\square_{2}\right)$}

In this appendix, we give the details of the computation of the imaginary part $\operatorname{Im}\left(\square_{(2)}\right)=M^{ \pm}$(see eq. (17)) 
of the operator

$$
\square_{(2)}=v_{F} V^{ \pm \dagger} \cdot\left(\alpha \sigma_{x} \frac{\partial V^{ \pm}}{\partial x}+\sigma_{y} \frac{\partial V^{ \pm}}{\partial y}\right) .
$$

Here

$$
\begin{aligned}
V^{+}(\mathbf{p}, \mathbf{r})= & \frac{\left(\begin{array}{c}
m(\mathbf{r}) v_{F}^{2}+\epsilon(\mathbf{p}, \mathbf{r}) \\
v_{F}\left(\alpha \Pi_{x}+i \Pi_{y}\right)
\end{array}\right)}{\sqrt{2(\epsilon(\mathbf{p}, \mathbf{r}))\left(m(\mathbf{r}) v_{F}^{2}+\epsilon(\mathbf{p}, \mathbf{r})\right)}} \\
V^{-}(\mathbf{p}, \mathbf{r})= & \frac{\left(\begin{array}{c}
v_{F}\left(\alpha \Pi_{x}-i \Pi_{y}\right) \\
-\left(m(\mathbf{r}) v_{F}^{2}+\epsilon(\mathbf{p}, \mathbf{r})\right)
\end{array}\right)}{\sqrt{2(\epsilon(\mathbf{p}, \mathbf{r}))\left(m(\mathbf{r}) v_{F}^{2}+\epsilon(\mathbf{p}, \mathbf{r})\right)}}
\end{aligned}
$$

are the normalized eigenvectors of the classical Hamiltonian $H^{ \pm}$(see Eq. (9)),

$$
\epsilon(\mathbf{p}, \mathbf{r})=H^{+}(\mathbf{p}, \mathbf{r})-U(\mathbf{r})=\sqrt{m(\mathbf{r})^{2} v_{F}^{4}+v_{F}^{2} \mathbf{\Pi}^{2}}
$$

and, with respect to spatial derivation, it is understood that $V^{ \pm} \equiv V^{ \pm}\left[\left(\partial S^{ \pm} / \partial \mathbf{r}\right), \mathbf{r}\right]$.

We perform here the calculation for $\mathrm{M}^{+}$, the one for $M^{-}$being essentially identical. We have

$$
\begin{aligned}
\alpha \operatorname{Im}\left[V^{+\dagger} \sigma_{x}\left(\partial_{x} V^{+}\right)\right] & =\frac{\alpha}{2 \epsilon\left(m v_{F}^{2}+\epsilon\right)} \operatorname{Im}\left[\left(m v_{F}^{2}+\epsilon, v_{F}\left(\alpha \Pi_{x}-i \Pi_{y}\right)\right) \cdot \sigma_{x} \cdot\left(\begin{array}{c}
\partial_{x}\left(m v_{F}^{2}+\epsilon\right) \\
v_{F} \partial_{x}\left(\alpha \Pi_{x}+i \Pi_{y}\right)
\end{array}\right)\right] \\
& =\frac{\alpha v_{F}}{2 \epsilon\left(m v_{F}^{2}+\epsilon\right)}\left[\left(m v_{F}^{2}+\epsilon\right) \partial_{x} \Pi_{y}-\Pi_{y} \partial_{x}\left(m v_{F}^{2}+\epsilon\right)\right]
\end{aligned}
$$

and in the same way

$$
\begin{aligned}
\operatorname{Im}\left[V^{+\dagger} \sigma_{y}\left(\partial_{y} V^{+}\right)\right] & =\frac{1}{2 \epsilon\left(m v_{F}^{2}+\epsilon\right)} \operatorname{Im}\left[\left(m v_{F}^{2}+\epsilon, v_{F}\left(\alpha \Pi_{x}-i \Pi_{y}\right)\right) \cdot \sigma_{y} \cdot\left(\begin{array}{c}
\partial_{y}\left(m v_{F}^{2}+\epsilon\right) \\
v_{F} \partial_{y}\left(\alpha \Pi_{x}+i \Pi_{y}\right)
\end{array}\right)\right] \\
& =\frac{\alpha v_{F}}{2 \epsilon\left(m v_{F}^{2}+\epsilon\right)}\left[\left(m v_{F}^{2}+\epsilon\right)\left(-\partial_{y} \Pi_{x}\right)+\Pi_{x} \partial_{y}\left(m v_{F}^{2}+\epsilon\right)\right]
\end{aligned}
$$

so that

$$
\operatorname{Im}\left(\square_{(2)}\right)=\frac{\alpha v_{F}^{2}}{2 \epsilon}\left(\frac{\partial}{\partial \mathbf{r}} \times \boldsymbol{\Pi}+\frac{\boldsymbol{\Pi} \times \partial_{\mathbf{r}}\left(m v_{F}^{2}+\epsilon\right)}{m v_{F}^{2}+\epsilon}\right) \cdot \mathbf{e}_{z},
$$

with $\mathbf{e}_{z}$ the unit vector in the direction perpendicular to the graphene plane.

Using finally that

$$
\begin{aligned}
& \left(\frac{\partial}{\partial \mathbf{r}} \times \mathbf{A}\right)_{z}=B \\
& \left(\partial_{\mathbf{r}} \times \partial_{\mathbf{r}} S\right)_{z}=\partial_{x} \partial_{y} S-\partial_{y} \partial_{x} S=0
\end{aligned}
$$

and that the Hamilton-Jacobi equation $E-$ $H^{+}\left(\frac{\partial S^{+}}{\partial \mathbf{r}}, \mathbf{r}\right)=0$ implies

$$
\frac{\partial}{\partial \mathbf{r}}\left(\epsilon\left(\frac{\partial S^{+}}{\partial \mathbf{r}}, \mathbf{r}\right)\right)=-\frac{\partial U}{\partial \mathbf{r}}
$$

then gives Eq. (18).

\section{APPENDIX B: GUIDING CENTER COORDINATES}

We sketch here the construction of the new canonical variables $\left(\mathbf{R}=(X, Y), \mathbf{P}=\left(P_{x}, P_{y}\right)\right)$ introduced in section IV C We start first by performing the simple canonical transformation $\mathbf{r} \rightarrow \mathbf{r}^{\prime}=\left(x, p_{y}\right), \mathbf{p} \rightarrow \mathbf{p}^{\prime}=\left(p_{x},-y\right)$.
Then, introducing the guiding center $\mathbf{r}_{0}=\left(x_{0}, y_{0}\right)$ coordinates

$$
\begin{aligned}
& x_{0}=x-\frac{1}{e B} \Pi_{y}=\frac{x}{2}-\frac{1}{e B} p_{y} \\
& y_{0}=y+\frac{1}{e B} \Pi_{x}=\frac{y}{2}+\frac{1}{e B} p_{x}
\end{aligned}
$$

we define the point transformation $\mathbf{R}\left(\mathbf{r}^{\prime}\right)$ as

$$
\mathbf{R}=\left(\frac{x}{2}+\frac{1}{e B} p_{y}, \frac{x}{2}-\frac{1}{e B} p_{y}\right)=\left(\frac{1}{e B} \Pi_{y}, x_{0}\right) .
$$

This transformation is obtained from the generating function $F\left(\mathbf{r}^{\prime}, \mathbf{P}\right)=\mathbf{P} \cdot \mathbf{R}\left(\mathbf{r}^{\prime}\right)$, and therefore the new momentum is given by

$$
\mathbf{p}^{\prime}=\frac{\partial F}{\partial \mathbf{r}^{\prime}}=\left(\begin{array}{c}
\frac{1}{2}\left(P_{x}+P_{y}\right) \\
\frac{1}{e B}\left(P_{x}-P_{y}\right)
\end{array}\right)
$$

which is easily inverted into

$$
\mathbf{P}\left(\mathbf{p}^{\prime}\right)=\left(p_{x}-\frac{e B}{2} y, p_{x}+\frac{e B}{2} y\right)=\left(\Pi_{x}, e B y_{0}\right) .
$$

The unperturbed Hamiltonian is then given as $H^{+}(\mathbf{p}, \mathbf{r})=v_{F}|\mathbf{\Pi}|=v_{F} \sqrt{P_{x}^{2}+(e B X)^{2}}$. 
[1] K. S. Novoselov, A. K. Geim, S. V. Morozov, D. Jiang, M. I. Katsnelson, I. V. Grigorieva, S. V. Dubonos, and A. A. Firsov, Nature 438, 197 (2005).

[2] A. H. Castro Neto, F. Guinea, N. M. R. Peres, K. S. Novoselov, and A. K. Geim, Rev. Mod. Phys. (2007).

[3] K. S. Novoselov, A. K. Geim, S. V. Morozov, D. Jiang, Y. Zhang, S. V. Dubonos, I. V. Grigorieva, and A. A. Firsov, Science 306, 666 (2004).

[4] C. Berger, Z. Song, T. Li, X. Li, A. Y. Ogbazghi, R. Feng, Z. Dai, A. N. Marchenkov, E. H. Conrad, P. N. First, and W. A. de Heer, J. Phys. Chem. 108, 19912 (2004).

[5] Y. Zhang, Y.-W. Tan, H. L. Stormer, and P. Kim, Nature 438, 201 (2005).

[6] T. Ando, T. Nakanishi, and R. Saito, Journal of the Physical Society of Japan 67, 2857 (1998).

[7] G. P. Mikitik and Y. V. Sharlai, Phys. Rev. Lett. 82, 2147 (1999).

[8] J. Bolte and S. Keppeler, Ann. Phys. 274, 125 (1999).

[9] M. V. Berry, Proc. R. Soc. Lond. A 392, 45 (1984).

[10] P. R. Wallace, Phys. Rev. 71, 622 (1947).

[11] J. C. Slonczewski and P. R. Weiss, Phys. Rev. 109, 272 (1958).

[12] J. W. McClure, Phys. Rev. 104, 666 (1956).

[13] M. O. Goerbig (2006), unpublished.

[14] V. P. Maslov and M. V. Fedoriuk, Semiclassical Approximation in Quantum Mechanics (D. Reidel Publishing
Company, 1981).

[15] R. G. Littlejohn and W. G. Flynn, Phys. Rev. Lett. 66, 2839 (1991).

[16] R. G. Littlejohn and W. G. Flynn, Phys. Rev. A 44, 5239 (1991).

[17] E. McCann and V. I. Fal'ko, Phys. Rev. Lett. 96, 086805 (2006).

[18] M. C. Gutzwiller, J. Math. Phys. 12, 343 (1971).

[19] M. C. Gutzwiller, Chaos in Classical and Quantum Mechanics (Springer-Verlag, 1990).

[20] S. C. Creagh, J. M. Robbins, and R. G. Littlejohn, Phys. Rev. A 42, 1907 (1990).

[21] K. Richter, D. Ullmo, and R. A. Jalabert, Phys. Rep. 276, 1 (1996).

[22] S. Keppeler, Phys. Rev. Lett. 89, 210405 (2002).

[23] S. Keppeler, Spinning Particles - Semiclassics and Spectral Statistics (Springer, 2003).

[24] P. Plochocka, C. Faugeras, M. Orlita, M. L. Sadowski, G. Martinez, M. Potemski, M. O. Goerbig, J. N. Fuchs, C. Berger, and W. A. de Heer, Probing the high-energy limit of massless dirac fermions in multi-layer graphene (2007)

[25] F. D. M. Haldane, Phys. Rev. Lett. 61, 2015 (1988).

[26] V. P. Gusynin and S. G. Sharapov, Physical Review B 73, 245411 (2006). 